\title{
Freeform Electronics for Advanced Healthcare
}

\author{
Muhammad M. Hussain, ${ }^{1, *}$ Aftab M. Hussain, ${ }^{1}$ Joanna M. Nassar, ${ }^{1}$ Arwa T. Kutbee, ${ }^{1}$ Abdurrahman Gumus, ${ }^{1}$ and \\ Amir N. Hanna ${ }^{1}$ \\ ${ }^{1}$ Integrated Nanotechnology Lab and Integrated Disruptive Electronic Applications (IDEA) Lab \\ Electrical Engineering, Computer Electrical Mathematical Science and Engineering Division, King Abdullah University of \\ Science and Technology (KAUST) \\ Thuwal 23955-6900, Saudi Arabia \\ *muhammad.hussain@kaust.edu.sa
}

\begin{abstract}
Freeform (physically flexible, stretchable and reconfigurable) electronics can be critical enabler for advanced personalized healthcare. With increased global population and extended average lifetime of mankind, it is more important than ever to integrate advanced electronics into our daily life for advanced personalized healthcare. In this paper, we discuss some critical criteria to design such electronics with enabling applications.
\end{abstract}

Index Terms - Freeform, electronics, healthcare.

\section{INTRODUCTION}

We live in a complex world with rising global population. While human resource can be a great asset but we first have to ensure they are healthy and happy. Interestingly rising global population imposes a challenge on further distribution of limited resources to ensure such healthy and happy life. Specially with more aging population with extended lifetime, we must have to ensure everyone leads a healthy and happy life. In that regard, electronics may potentially be used to facilitate such prosperity. It is true that today's electronics are sufficiently powerful in context of data management (processing, storage, communication, display). However, they are often rigid, bulky, brittle and uniformly structured. Interestingly, human bodies have more irregularity in their shapes, asymmetry on their skins and inherent softness. Therefore, a critical barrier for seamless integration of today's advanced electronics with human body lies in their interfaces. One potential solution is to use soft curvilinear electronics which can be easily conformed to human body with suitable mechanical dexterity. From that perspective, in the last two decades, many interesting exciting materials have been emerged fuelled by innovation in chemistry and material science disciplines [1]. While organic and molecular electronics are spearheading and have penetrated in the high resolution display market, their inherent limited electron transport characteristics, thermal instability and ultra-largescale-integration (ULSI) incompatibility, impede their future and wide ranging success. One dimensional material such as carbon nanotube (CNT) and various nanowires overcome the electron transport and thermal instability issue with great prowess but largely fail due to the ULSI incompatibility. While two dimensional graphene or other dichalcogenide atomic crystal structure materials are promising, still their manufacturability is a valid and sustained concern for the integrated device manufacturers (IDMs). Therefore, with due credits to all these emerging materials, traditional crystalline materials like silicon, silicon germanium, gallium nitride, III-V materials based electronics can still be the backbone of freeform electronics as long as we can make them physically flexible, stretchable and reconfigurable. Therefore, in this paper we will describe some important criteria for such electronic system design focusing on advanced healthcare for both in-vivo and in-vitro applications. We will focus on bulk monocrystalline silicon (100) which is used to fabricate ninety percent of the electronics today.

\section{DESIGN CRITERIA FOR FREEFORM ELECTRONICS FOCUSING ON ADVANCED HEALTHCARE APPLICATIONS}

First and the foremost challenge is the choice of biocompatible materials based electronics whose integration density and performance plus energy efficiency is strictly directed by thermal compatibility of human body. It is well known that human body (both in-vivo and in-vitro) should not experience more than $2^{\circ} \mathrm{C} / \mathrm{cm}^{2}$ heat increment. This dictates that the associated power consumption from body integrated electronics is restricted to $40 \mathrm{~mW} / \mathrm{cm}^{2}$. Now, take an example of brain mapping. According to the World Health Organization (WHO), twenty five percent of world's population suffer from some sort of neurological disorders. Examples can be from normally ignored depression to Alzheimer's disease, Parkinson's disease, etc. Therefore, it is important to study (map) our brain activities. Today's gold standard is microneedle based Utah Array which is the most effective method as of today but after a yearlong constant use, it inflames the penetration area and needs immediate removal. Thus makes it an invasive system. Non-invasive systems such as Magnetic Resonance Imaging (MRI) can be fantastic, however, it's humongous size and weight with kilowatts range power consumption makes it unsuitable for constant use. Also, when an episode begins for a patient, unless we have a convenient wearable system, it becomes undeniably difficult to monitor brain activity. Now, electroencephalogram (EEG) can be used as non-invasive wearable system, however data acquisition through such system comes with very weak signal-to-noise ratio and thus making the overall reliable data crunch nearly impossible. Hence, if we can make a soft patch which will be placed on brain's soft cells and tissues in the intracranial space, then it can possibly send us invaluable information. Now, the challenge begins as human brains have 82 billion neurons. It is impossible that every single of them can be tracked in foreseeable future. At the same time, brain's soft exposed areas for exploration is limited to mere $8 \times 8 \mathrm{~cm}^{2}$ area. Therefore, it is important for us to use every bit of that area with soft sensors. However, sensors collecting analog signals need associated minimal electronics to convert the data into digital signals and then transmitting that to out of scalp. Hence, if we follow traditional integration schemes of lateral in-plane monolithic integration, it is impossible to maximize number of sensors within a limited space. Therefore, recently we have demonstrated a complementary metal oxide semiconductor (CMOS) based integration strategy which allows soft materials like polymer to be used as interposing base layer and 
fabricating soft biocompatible sensors on one side and relevant data management electronics on the other side (Fig. 1) [2]. An effective analogy will be two sides of the same coin. However, it is easy to say than done. Although it sounds like 3D-IC design, in reality having a soft medium makes the integration sequence highly complex. Still, using electrochemical deposition we overcame the challenge with through-polymer-via (TPV) formation. These highly conductive copper TPVs ensure seamless flow of electrical signals from the sensors on one side to processing electronics on the other. We utilized state-of-theart electronics fabrication processes to ensure compatibility with high-end electronics. Further, the scalable fabrication process ensures the successive miniaturization of sensor nodes with generation of the device, thus providing higher spatial resolution. We enhanced the integration scheme by integrating multiple layers of electronics on the sensor array to obtain a completely integrated area-efficient system for Electrocorticography (ECoG). Hence, we demonstrated the integration of sensor array within a soft material system (polyimide), with state-of-the-art silicon CMOS circuitry. Further, the integration scheme can be used to integrate layers different materials together making heterogeneous 3D integration possible. The resulting system is free-form (flexible and stretchable) if the component layers are free-form. With this integration strategy, going forward we will be able to design body integrated electronics (both in-vivo and in-vitro) in area efficient manner.

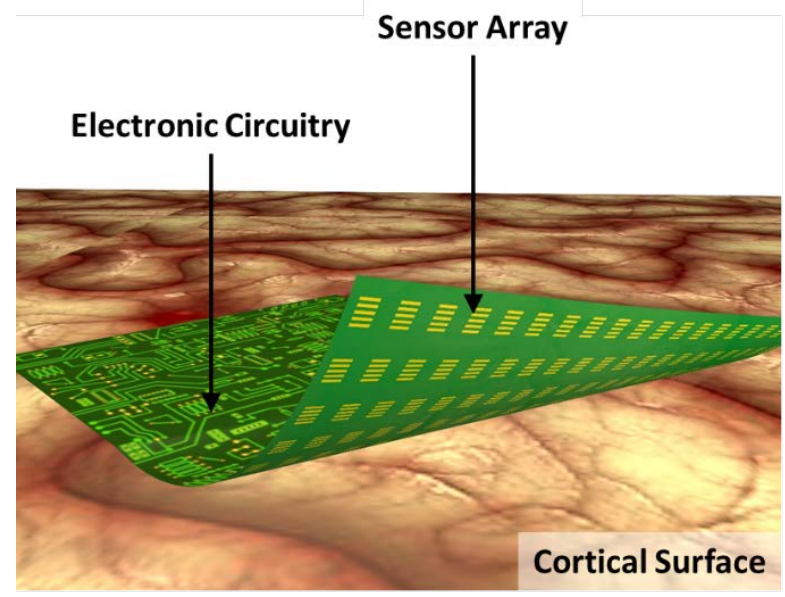

Fig. 1. Illustration of a flexible, 3D integrated brain machine interface.

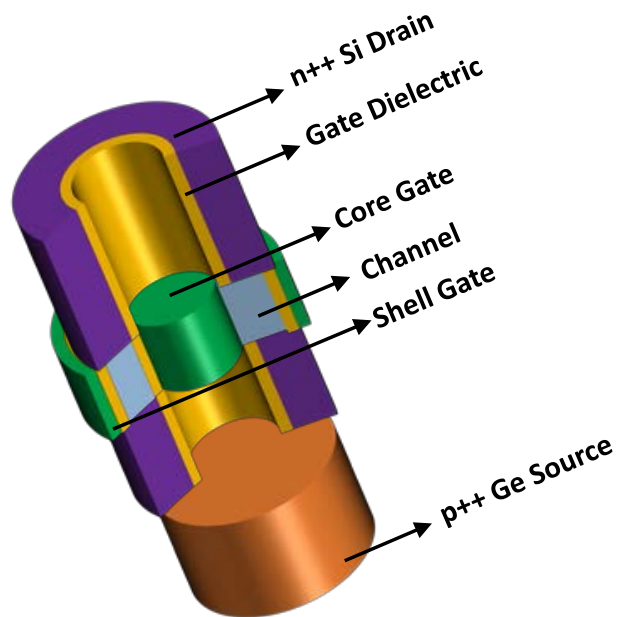

Fig. 2. Illustration of a hetero-structure nanotube TFET with core-shell gates.

Next challenge is thermal management. State-of-the-art planar and non-planar transistors have an established trend of area scaling resulting into higher power dissipation induced hotter electronics and systems. To address this critical issue, we have designed a nanotube architecture based heterogeneous tunnel field effect transistor (TFET) with core-shell gate stacks (Fig. 2) [3, 4]. Such device offers the highest performance (drive current) among all the reported TFETs with pinned subthreshold slope lesser than $60 \mathrm{mV} / \mathrm{dec}$. Such device overcomes issues of paradoxical relationship of limited performance and lower power dissipation in traditional devices. By using heterostructure materials system we have taken advantage of higher injection from source side and thus higher drain current.

After integration strategy and thermal management, important consideration is the operating environment which is bio-fluidic or skin-mounted. In both cases, conventional radio frequency (RF) antenna based communication medium fails due to unintended frequency dispersion and attenuation. Therefore, from antenna design to their integration and choice of material has to sustain within bio-fluidic environment which is considered as lossy medium. For skin-mounted wearables, it if affected by anomalous physical deformation related to our daily activities such as flexing, stretching, twisting, etc. Therefore, not only we need to develop ultra-thin flexible antenna, but also a stretchable one. In the past several in-plane stretchable antennas have been demonstrated but due to stretching their length fundamentally changes which compromises their fixed frequency operation. Therefore, we adopted a design of helical architecture which stretches out-ofplane and its core body remains detached from the skin, lowering lossy skin medium's interference (Fig. 3) [5]. Additionally, using copper (which is biocompatible) as based metal for the antenna, which is not physically stretchable, we overcame the issue of permanent physical elongation based frequency dispersion and attenuation. However, due to fractal design, copper based helical architecture antenna allowed to have sufficient stretchability to withstand daily activities of human body. Using such novel but balanced integration strategy, we demonstrated wearable antennas which is operable even up to 390 meters at fixed frequency of $2.4 \mathrm{GHz}$.

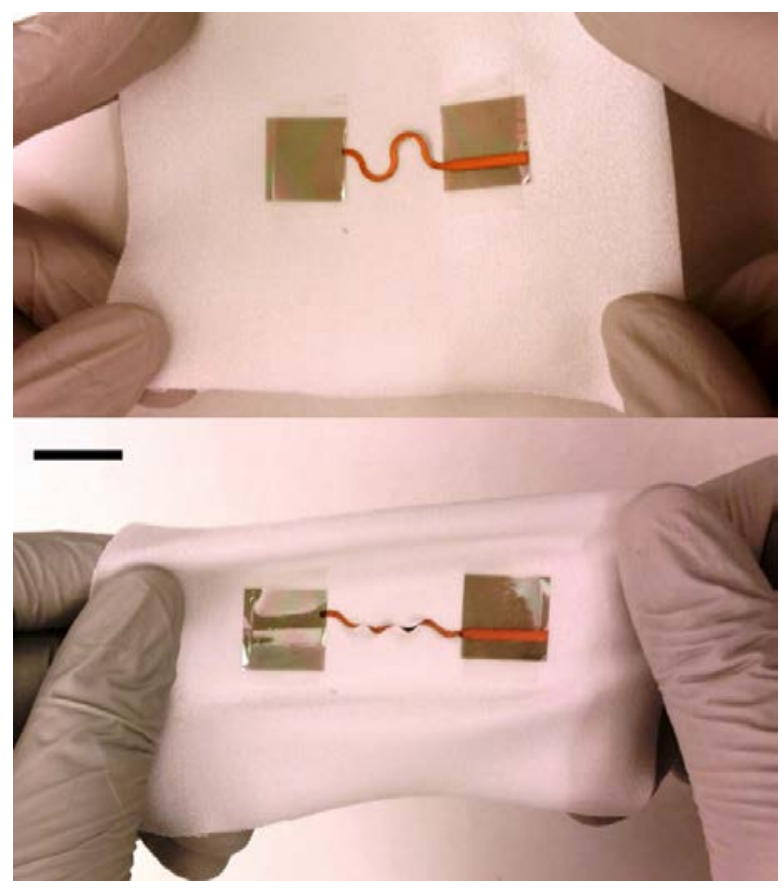

Fig. 3. Optical images of fabricated antenna attached to a stretchable fabric, before and after $30 \%$ straining. Scale bar is $2 \mathrm{~cm}$. 
Next important consideration is power management. While it is important to lower power consumption of any electronics in a world of growing number of electronics, it is equally important to have biocompatible energy harvesting and storage system. In that regard, we have unusual energy resource like bio-fluids such as sweat and saliva. We redesigned microbial fuel cells (MFCs) using CMOS compatible processes and achieved $\mathrm{nW}$ to $\mathrm{mW}$ level power generation. Now, what is MFC? Although nearly eighty percent of the earth has water, only a limited percentile is potable. Reverse osmosis is the leading technology among seven other corresponding technologies but each of them is excessively power consuming. Therefore, water-energy nexus is a critical challenge in today's world with rising population. MFC is a lab based interesting device whose electrogenic bacteria can consume waste from the waste water and in return releases electrons. If properly collected, such electrons can make the overall cell operation sustainable and energy independent. Thus a MFC can purify water while saving precious energy. We deployed nickel monosilicde based Ohmic contact and silicon based CMOS technology to develop micro-scale MFC which can generate $\mathrm{mW}$ level of power for on-chip power generation. By using saliva as fuel, we demonstrated $\mathrm{nW}$ scale power generation with further potential of scaling up [6]. More importantly, by doing so we have eliminated status-quo fuels such as chemically hazardous acetate based chemistry. Additionally, by removing traditional proton exchange membrane, we have made the overall device affordable. Therefore, such devices are important for in-vivo power generation with bio-compatibility.

Final criteria for any device for wider deployment to benefit the larger portion of the world's population is economy. While CMOS technology can certainly drive down the cost, but CMOS technology alone cannot do it for every person on earth. Therefore, recently we have demonstrated household materials based wearable multi-functional skin-type flexible integrated platform which can monitor the environment with out-of-plane arrangement and body vitals with in-plane integration [7]. A comparative study shows that such wearable gadgets provide the body vitals like body temperature, heartrate, blood pressure and skin hydration data same as that of obtained from smart watches (Apple Watch and Samsung Gear) within $\pm 5 \%$ accuracy but significantly lower cost $(<\$ 25)$. Now, how is it even possible to use household materials for such sophisticated functionalities? Oddly enough, most physical sensing is dependent on resistive and/or capacitive sensing. Now, both of these sensing really depend on conductive and insulating materials. Our investigative study shows household materials such as aluminium foils, copper foils, plastic wraps, sponge, $3 \mathrm{M}$ papers, etc. with their conductive medium and micro-fibril architecture are effective conductive and resistive materials. Next, their capacitive and resistive properties can be further tuned based on their shapes and sizes and that is exactly how we built variety of sensors: pressure, proximity, temperature, humidity, flow, photo, strain sensors, etc. Another beauty of the overall scheme is paper has been in use for hundreds of years and such sensors can be made easily with record affordability. This also perfectly aligns with our vision of Do It Yourself (DIY) Electronics - simple to understand and learn and easy to implement and use electronics.

\section{KEY ADVANCED HEALTHCARE APPLICATIONS}

We will briefly discuss three wearable freeform electronic systems which essentially disrupt the way today's healthcare technologies and ways. Our first example is smart thermal patch. Millions of world's population suffer from daily bruises, pains, arthritis and such. Most often, such suffering population use chemical based off-the-shelves patches which often are not suitable for children. For various locations of human body, we have to use different sizes raising the economic consideration. And finally, they can work at the most for ten hours only and then driving the cost up further. Therefore, we have demonstrated a copper (any biocompatible, corrosion resistant, economical material with suitable residual stress can be used) based CMOS compatible integration strategy to develop a smart digitized thermal patch integrating copper based heaters and temperature sensors which can conform to irregular body contours (Fig. 4) [8]. Moreover the whole system is stretchable up to $300 \%$ thus such a system can be used while wrapping our index fingers and then can be stretched out to cover larger space of other body locations. Such flexibility and stretchability is unprecedented. With appropriate integration of freeform silicon CMOS electronics, we have demonstrated App based smart gadget controlled pain management. In future, such system can be used for precision thermotherapy based targeted destruction of cancerous cell instead of conventional chemotherapy with negative side effects.

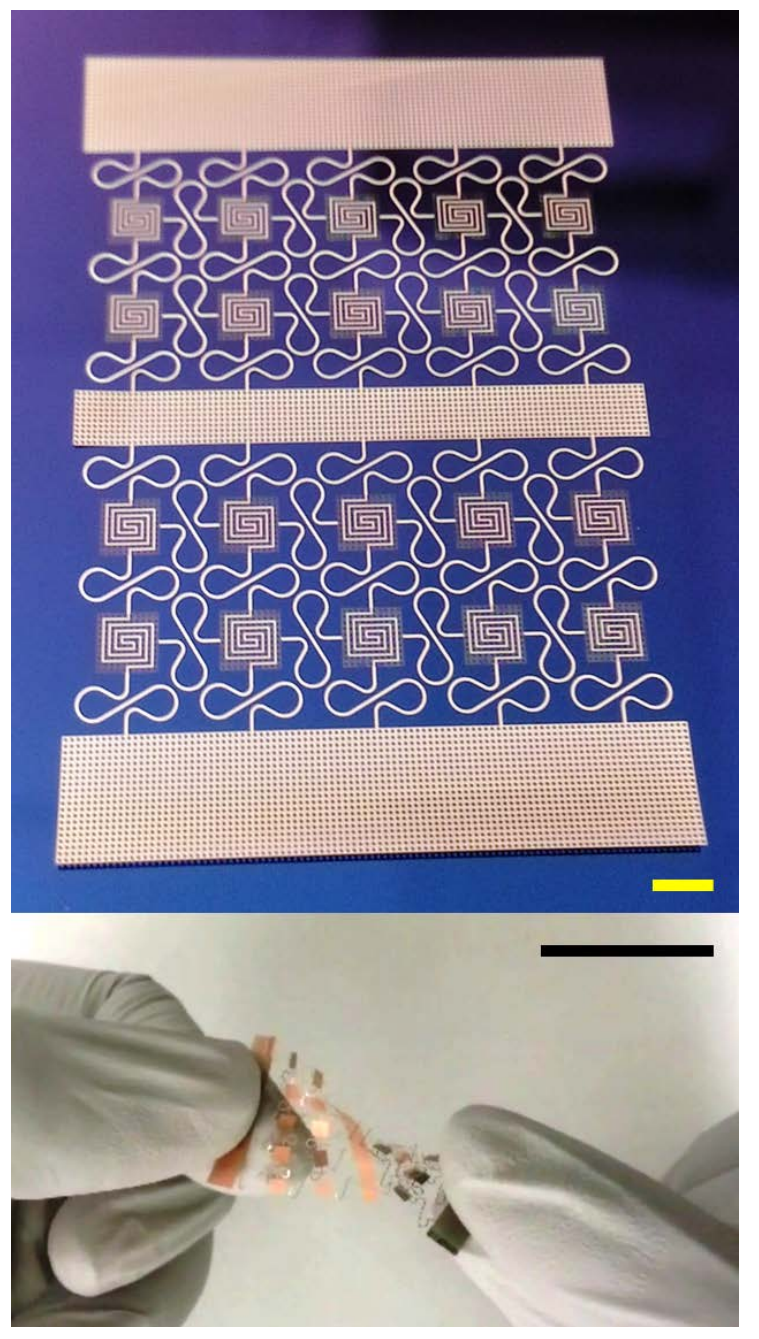

Fig. 4. Optical images of fabricated thermal patch on the wafer, and being handled with bare hands without the use of special handling equipment. Scale bars are $2 \mathrm{~mm}$ and $2 \mathrm{~cm}$ respectively.

Our next example is presumably world's first wearable invivo device. Today for orthodontic treatment, metallic one-sizefits-all dental braces are used for bon regeneration. It costs 
thousands of dollars associated with its lingering usage time. With the advances in panoramic x-ray technology, we are now capable to personalize such dental braces by making them using additive manufacturing such as 3D printing (even using transparent materials). We have taken more advanced steps by integrating near-infrared lasing capability embedded on and in this personalized 3D printed transparent braces. Such capability enhances the bone regeneration significantly and reduces the overall cost and discomfort. However, it would not have been possible without an appropriate energy storage. Major challenge to integrate any traditional lithium based energy storage is their toxicity. Therefore, we have introduced nontoxic micro-scale flexible batteries to be used as on-demand power supply. In future we envision to use smart phone based remote (wireless) powering for such in-vivo devices for advanced healthcare applications.

Our final example is inspired by the vision, "What is after sensors?” Today more and more we are embracing Internet of Things (IoTs) which suggests trillions of sensors deployed worldwide to create cyber-physical system which eventually generates big data. Personalized healthcare initiatives are geared toward developing understanding about personalized healthcare need (instead of blanket globalized treatment) by analyzing genetic mapping by chip-based genome sequencing and physiological and psychological monitoring using wearables. However, going forward in future when we enter the era of Internet of Everything (IoE), the main question would be what we do with the analysed big data. To address such questions, we have taken steps to personalize nutrient formulation and eventually personalize medicines, by developing wearable personalized medicinal platform. Using expendable polymer based smart microfluidics architecture and freeform CMOS electronics, we have shown that based on an already established library of genetic mapping and instant collection of physiological and psychological data, we can dynamically formulate the required nutrition level or medicine of a user, prepare it in-situ and deliver on-demand. Such devices are unprecedented and shows the realization of the vision the future of affordable advanced personalized healthcare for everyone.

\section{CONCLUSIONS}

In this paper, we have laid down our vision about freeform electronics for advanced personalized healthcare. We have shown five main design criteria for any such device (in-vivo and in-vitro): (i) integration strategy, (ii) thermal management, (iii) biocompatibility, (iv) power management and (v) economics. By using examples ranging from brain machine interface to low-cost paper-based wearable monitors we have shown criticality of such design criteria. Further, we have presented three advanced personalized healthcare freeform electronic systems which will disrupt the way we know and use healthcare today. Presented devices are smart digitized thermal patch for pain management, smart dental brace for accelerated orthodontic treatment and finally wearable personalized medicinal platform for in-situ dynamic formulation and ondemand delivery of nutrients and medicines. Immense possibilities exist going forward to positively change the way we lead our life today and multi-disciplinary effort with open minded engineering innovation, we can accelerate such growth to bring tomorrow's world to today.

\section{ACKNOWLEDGMENT}

This publication is based upon work supported by the King Abdullah University of Science and Technology (KAUST) and Boeing Company.

\section{REFERENCES}

[1] A. M. Hussain and M. M. Hussain, "CMOS-Technology-Enabled Flexible and Stretchable Electronics for Internet of Everything Applications,” Adv. Mater., vol. 28(22), pp. 4219-4249, Jun. 2008.

[2] A. M. Hussain and M. M. Hussain, "Deterministic Integration of Outof-Plane Sensor Arrays for Flexible Electronic Applications,” Small, vol. 12(37), pp. 5141-5145, Oct. 2016.

[3] A. N. Hanna, H. M. Fahad and M. M. Hussain, "InAs/Si Hetero-Junction Nanotube Tunnel Transistors,” Sci. Rep. vol. 5, 9843, doi:10.1038/srep09843, 2015.

[4] A. N. Hanna and M. M. Hussain, "Si/Ge Hetero-structure Nanotube Tunnel Field Effect Transistor,” J. Appl. Phys. vol. 117, pp. 014310, Jan. 2015.

[5] A. M. Hussain, F. A. Ghaffar, S. I. Park, J. A. Rogers, A. Shamim and M. M. Hussain, "Metal/Polymer Based Stretchable Antenna for Constant Frequency Far-Field Communication in Wearable Electronics," Adv. Funct. Mater. vol. 25(42), pp. 6565-6575, Nov. 2015.

[6] J. E. Mink, R. Qaisi, B. E. Logan and M. M. Hussain, "Energy Harvesting from Saliva in Micro-Sized Microbial Fuel Cells,” NPG (Nature) Asia Mater. vol. 6, pp. e89, Mar. 2014.

[7] J. M. Nassar, M. D. Cordero, A. T. Kutbee, M. A. Karimi, G. A. Torres Sevilla, A. M. Hussain, A. Shamim and M. M. Hussain, "Paper Skin Multi-Sensory Platform for Simultaneous Environmental Monitoring,” Adv. Mater. Tech. vol. 1(1), Apr. 2016.

[8] A. M. Hussain, E. B. Lizardo, G. A. Torres Sevilla, J. M. Nassar and M. M. Hussain, "Ultra-Stretchable and Flexible Copper Interconnect Based Smart Patch for Adaptive Thermotherapy,” Adv. Healthcare Mater. vol. 4(5), pp. 665-673, Apr. 2015. 